\title{
Vers une accentuation des disparités dans le financement de l'agriculture en Afrique de l'Ouest ?
}

\author{
Vincent Ribier* et Jean-Jacques Gabas \\ CIRAD, UMR ART-DEV, 45 bis, avenue de la Belle-Gabrielle, 94736 Nogent-sur-Marne cedex, France
}

\begin{abstract}
Résumé - Le paysage institutionnel du financement de l'agriculture est en cours de recomposition, avec la multiplication et la diversification des acteurs, ainsi que l'imbrication croissante des financements publics et privés. La volonté d'attirer de nouveaux opérateurs privés se traduit par la mise en place de montages financiers où les fonds publics sont censés jouer un effet de levier. Cette évolution interroge la capacité des États à cibler les financements pour répondre aux grands enjeux de développement tels que la sécurité alimentaire, la réduction de la pauvreté et la modernisation de l'agriculture. Une étude comparative menée dans trois pays d'Afrique de l'Ouest (Sénégal, Côte d'Ivoire, Ghana) apporte des éléments de réponse. Les institutions nationales sont généralement trop faibles pour canaliser les nouveaux flux de financement vers les objectifs affichés de la politique agricole. Les disparités de financement du secteur agricole, déjà marquées par le passé, s'en trouvent exacerbées et l'accroissement global des montants de financement s'accompagne de la concentration de ceux-ci au profit de l'agriculture entrepreneuriale et au détriment de l'agriculture familiale, des biens publics et de certaines régions enclavées.
\end{abstract}

Mots clés : financement / agriculture / pilotage / ciblage / disparités / Afrique de l'Ouest

\begin{abstract}
Increasing disparities in agricultural funding in West Africa? The new architecture of the development financing is in the way of reorganization, marked by the multiplication and the diversification of the actors and the growing interweaving of the public and private financing. The will to attract new private operators leads to the implementation of financial mechanisms where the public funds are supposed to play leverage. This evolution questions the capacity of African States to address the big issues of development such as food security, reduction of poverty and modernization of agriculture. A comparative study in three West African countries (Senegal, Ivory Coast, and Ghana) provides some answers. The national institutions are generally too weak to channel the new flows of financing towards the agricultural policy objectives. The disparities of financing of the farming sector, already marked in the past, are aggravated there and the global increase of the amounts of financing comes along with the concentration of these for the benefit of the entrepreneurial farmers and to the detriment of family farmers, of public goods and of certain enclosed regions.
\end{abstract}

Keywords: financing / agriculture / piloting / targeting / disparities / West Africa

\section{La recomposition du paysage institutionnel des financements}

Le secteur agricole connaît un regain d'intérêt au niveau international depuis la crise alimentaire de 2008, après une marginalisation qui s'était affirmée au milieu de la décennie 1980. L'année de l'agriculture familiale décrétée par les Nations Unies pour 2014, ou encore la place donnée au secteur dans les objectifs de développement durable énoncés fin 2015, confirment ce regain d'intérêt. On observe dans ce contexte un accroissement global des flux de financement de l'agriculture, mais l'évolution la plus remarquable est sans nul doute la

\footnotetext{
* Auteur de correspondance : vincent.ribier@cirad.fr
}

modification de la structure des financements, avec la montée en puissance de nouveaux acteurs, tant publics que privés, ainsi que la mise en place de divers dispositifs institutionnels destinés à attirer et à canaliser les nouveaux financements.

Le paysage institutionnel du financement de l'agriculture est en cours de recomposition. Les bailleurs de fonds traditionnels du Comité d'aide au développement de l'Organisation de coopération et de développement économiques (CAD/OCDE), soumis à des contraintes budgétaires, voient baisser leur importance relative dans le financement de l'agriculture au profit de nouveaux acteurs : fondations privées (type Gates, Danone Écosystème), nouveaux fonds portés par des entreprises privées (livelihoods fund), acteurs venant des pays émergents (Chine, Inde, Brésil notamment), fonds d'investissements privés qui se positionnent sur le secteur 
Tableau 1. Structure et évolution du financement du secteur agricole de trois pays d'Afrique de l'Ouest (en millions $\$$ US, moyenne annuelle sur chacune des deux périodes).

Table 1. Structure and evolution of agricultural funding in three West African countries (in millions of US\$, annual average on both periods).

\begin{tabular}{|c|c|c|c|c|c|c|}
\hline & \multicolumn{2}{|c|}{ Côte d'Ivoire } & \multicolumn{2}{|c|}{ Ghana } & \multicolumn{2}{|c|}{ Sénégal } \\
\hline Gouvernement & 135 & 185 & 528 & 643 & 163 & 181 \\
\hline Bailleurs de fonds & 46 & 68 & 135 & 149 & 125 & 156 \\
\hline Total financement & 432 & 600 & 1019 & 1297 & nd & 497 \\
\hline
\end{tabular}

Source : Gabas et Ribier, 2015.

agricole suite à la crise financière de 2007-2008 (Afrique contemporaine, 2011), fonds de garantie. Sans oublier les fonds issus de la diaspora, ceux venant des exploitants agricoles euxmêmes ainsi que les financements en provenance du secteur bancaire.

Cette recomposition est particulièrement perceptible sur le continent africain. Le Groupe des huit (G8) a lancé en 2012 la Nouvelle alliance pour la sécurité alimentaire et la nutrition (NASAN) en partenariat avec le Nouveau partenariat pour le développement de l'Afrique (NEPAD). Le NEPAD avait lancé au préalable, en lien avec les structures régionales telles que la Communauté économique des États d'Afrique de l'Ouest (CEDEAO), des initiatives destinées à mobiliser davantage de financements, dans le cadre de plans d'investissement agricole, qui se déclinent aux niveaux régional (PRIA) et national (PNIA). Dans tous les cas, l'objectif est d'accroître les financements de l'agriculture en s'efforçant d'attirer de nouveaux opérateurs privés dans le cadre de montages financiers où les fonds publics sont censés jouer un effet de levier (Orliange et de Milly, 2015).

La mobilisation croissante de ressources et les changements majeurs dans le paysage institutionnel du financement de l'agriculture sont a priori une «bonne nouvelle » pour les États dont les besoins financiers sont immenses du fait du manque d'intérêt porté à l'agriculture pendant près de 20 ans. Toutefois, cette évolution du paysage du financement affecte sa gouvernance et l'on peut s'interroger sur la cohérence entre la dynamique observée récemment et les politiques publiques agricoles mises en œuvre. L'enjeu de celles-ci est d'orienter ces financements croissants vers les cibles prioritaires pour un développement « inclusif », à savoir les agriculteurs familiaux, les jeunes ruraux qui arrivent massivement chaque année sur le marché du travail, mais aussi les biens et les services publics. Dans ce contexte relativement nouveau, l'architecture institutionnelle du financement de l'agriculture actuellement en cours de recomposition renforce-t-elle ou non les stratégies de ciblages ? Les États ont-ils la capacité de garder le contrôle de la situation et de canaliser les ressources vers un modèle de développement agricole « inclusif »? Pour apporter des éléments de réponse à ces questions, nous aborderons tour à tour les stratégies de financement des différents acteurs et la capacité des États à piloter et à orienter ces financements vers leurs objectifs prioritaires affichés.

\section{Des stratégies de ciblage des financements plus ou moins explicites selon les acteurs}

Les investisseurs privés, déjà largement présents dans certains pays d'Afrique de l'Ouest, tendent à accroître leur influence depuis ces dernières années, particulièrement dans les pays à revenu intermédiaire. Le montant de leurs investissements est ainsi nettement supérieur aux dépenses publiques agricoles dans le cas de la Côte d'Ivoire ; il s'en rapproche dans le cas du Ghana (Tab. 1). Cela est moins vrai pour les pays les moins avancés, où les dépenses publiques agricoles restent largement majoritaires, comme dans le cas du Sénégal. Diverses initiatives, destinées à attirer les investisseurs privés, pourraient toutefois faire évoluer cet état de fait dans les prochaines années.

L'évolution de la structure des financements est lourde de conséquences sur la dynamique du secteur agricole tant il est vrai que l'origine des financements influe sur la manière dont ceux-ci sont affectés en termes de destinataires, de filières et de régions. Chaque type d'acteur oriente ses flux de financement selon ses propres objectifs. Nous tentons ici une brève caractérisation des structures de financement par type d'acteurs à partir des données recueillies dans trois pays d'Afrique de l'Ouest, la Côte d'Ivoire, le Ghana et le Sénégal (Brochard, 2015 ; Gabas et Ribier, 2015 ; Varlet, 2015).

\subsection{Différence d'affectation des dépenses selon qu'elles proviennent des États ou des bailleurs}

Certaines récurrences sont observées dans l'affectation différentielle des dépenses entre les États et les bailleurs. Les dépenses de l'État financent en premier lieu le fonctionnement des administrations (notamment les salaires), les instances parapubliques, ainsi que les filières traditionnelles d'exportation telles que le cacao au Ghana, le café-cacao et le coton en Côte d'Ivoire ainsi que l'arachide au Sénégal. Les subventions aux intrants, élément majeur des dépenses publiques agricoles dans de nombreux États d'Afrique de l'Ouest, sont quasi exclusivement financées par des ressources internes. Les bailleurs sont quant à eux plus particulièrement positionnés sur le financement des infrastructures, notamment les périmètres 
hydro-agricoles (financés à $74 \%$ sur ressources externes en Côte d'Ivoire), le riz et plus généralement le secteur vivrier. Certains bailleurs tels que le Japon interviennent quasi exclusivement dans le secteur du riz.

\subsection{Des modalités différentes selon les bailleurs}

Chaque bailleur de fonds mobilise, selon son histoire et ses propres objectifs, certains outils plutôt que d'autres. D'une manière générale, les bailleurs bilatéraux privilégient les dons (au Sénégal, plus de $90 \%$ des financements extérieurs bilatéraux sont des dons), tandis que les bailleurs multilatéraux sont essentiellement positionnés sur des prêts. L'octroi d'aide budgétaire, modalité privilégiée par l'Union européenne et le Canada, est conditionné à l'atteinte de certains objectifs. L'Agence française de développement mixe dons et prêts. D'autres bailleurs privilégient des financements sous forme de fonds de garantie comme l'United States Agency for International Development (USAID) au Sénégal dans le cadre de son Projet croissance économique (PCE), qui s'adresse plus directement au secteur privé national. Les modalités, orientations, et procédures de ces multiples bailleurs sont en conséquence très différentes les unes des autres et la question de la cohérence d'ensemble peut être posée.

\subsection{La grande variété des financements privés}

Les financements privés sont très divers : autofinancement des producteurs, investissements des entreprises privées (nationales et internationales), transferts des migrants, financements informels des exploitants agricoles venant de voisins, de la famille ou d'amis, ou encore financements venant des institutions de microcrédits. Ces multiples formes de financements privés ne font pas l'objet d'un suivi rigoureux bien que leur rôle soit largement reconnu.

Il est communément admis que les agriculteurs sont les premiers financeurs du secteur agricole. Le rapport Investir dans l'agriculture pour un monde meilleur (FAO, 2012) souligne que, dans les pays à faible ou à moyen revenu, les investissements que les agriculteurs consacrent à l'équipement de leurs propres exploitations agricoles sont, chaque année, plus de quatre fois plus importants que les investissements des pouvoirs publics dans le secteur agricole, et notoirement supérieurs à ceux des donateurs internationaux et des investisseurs étrangers privés. Il est toutefois très difficile d'avoir une estimation, même approximative, du montant global de l'autofinancement des exploitations agricoles.

Les fonds d'investissements privés et les entreprises privilégient d'une manière générale les filières ayant une forte composante industrielle, comme le sucre et la banane en Côte d'Ivoire, ou encore l'hévéa et le palmier à huile. Au Sénégal, les données de l'Agence nationale pour la promotion de l'investissement et des grands travaux (APIX) montrent que les investissements privés étrangers se positionnent prioritairement sur l'agriculture d'exportation : $90 \%$ des investissements relevant du régime Entreprises franches d'exportation (EFE), et donc ciblés sur les marchés extérieurs, sont des Investissements directs étrangers (IDE).

Les rapatriements de revenus des migrants, bien que difficiles à évaluer avec précision, semblent conséquents. Ils sont évalués globalement par la Banque centrale des États d'Afrique de l'Ouest (BCEAO), mais la part consacrée à l'agriculture ne fait l'objet d'aucun suivi précis. En 2011, le gouvernement sénégalais estimait à 21 milliards de francs CFA le volume des transferts des migrants dirigés vers le secteur agricole national, soit approximativement le quart des dépenses agricoles de l'État. On peut supposer que l'agriculture familiale est le principal destinataire de ces transferts, sans être en mesure de préciser davantage la ventilation par filières et par régions.

De même, les montants globaux des crédits octroyés par les Institutions de microfinance (IMF) sont connus, sans que soit précisée la part consacrée au monde agricole. Sous l'hypothèse que $20 \%$ du total des crédits sont alloués à l'agriculture, les montants annuels mobilisés au Sénégal par la microfinance pour un usage agricole seraient de l'ordre de 45 milliards de francs CFA, soit le double des transferts agricoles des migrants et la moitié des dépenses publiques agricoles sur ressources internes.

\section{La difficile émergence de stratégies nationales de financement de l'agriculture}

La diversification des sources et des modalités de financement de l'agriculture rend d'autant plus nécessaire la mise en place de mécanismes de pilotage et d'orientation des différents flux pour atteindre les objectifs affichés d'amélioration de la sécurité alimentaire, de réduction de la pauvreté et de modernisation de l'agriculture. Cela suppose une capacité de leadership des pouvoirs publics, qui est dans la plupart des cas bien trop faible pour avoir un effet déterminant sur le ciblage des financements. Le PNIA, outil conçu et promu par le NEPAD et la CEDEAO comme cadre de cohérence pour l'élaboration des priorités budgétaires nationales, peine à remplir sa mission d'orientation des différentes sources de financement vers des objectifs communs (OXFAM, 2015).

Les PNIA recensent, sous un format relativement standard et donc comparable d'un pays à l'autre, les besoins de financement pour le secteur agricole de chaque pays (voir notamment République du Sénégal, 2010 ; République de Côte d'Ivoire, 2010 ; IPAR, 2010). Leur capacité de pilotage et d'orientation est toutefois limitée par la difficulté des États africains à imposer aux autres acteurs (bailleurs, investisseurs privés, fondations) ce cadre comme la référence incontournable en matière de financement. Ces derniers restent libres d'y avoir recours ou non au moment de cibler leurs propres financements, de telle sorte que les interventions recensées dans les PNIA s'apparentent davantage à un catalogue non hiérarchisé qu'à une stratégie nationale de financement agricole. En pratique, on observe des comportements très variables à l'égard des PNIA selon le type d'acteurs :

- les services gouvernementaux sont les acteurs ayant le plus directement recours au PNIA. Il n'est toutefois pas démontré que toutes les dépenses sur ressources internes soient faites sur la base des priorités affichées par les PNIA (manque de traçabilité des circuits de décaissement). La mise en place de nouveaux programmes (comme le Programme d'accélération de la cadence de l'agriculture sénégalaise [PRACAS] au Sénégal) brouille parfois l'image du PNIA en le faisant apparaître comme un document daté qui n'est plus la référence ; 
- les institutions parapubliques (par exemple du café-cacao en Côte d'Ivoire et du cacao au Ghana) montrent une autonomie certaine par rapport au PNIA quant à la détermination de leurs budgets et de leurs dépenses ;

- les bailleurs ont un recours parfois distant au PNIA. Ils établissent des programmes d'action pluriannuels avec les instances nationales, mais ils reconnaissent bien souvent en aparté qu'ils ne se réfèrent qu'occasionnellement au PNIA quand ils établissent leur portefeuille de projets et réalisent leurs décaissements. Leurs priorités obéissent à d'autres agendas et la référence au PNIA ne se fait le plus souvent qu'ex-post pour justifier de quelle manière leurs projets contribuent à l'atteinte des objectifs du document;

- les opérateurs privés, quant à eux, se soucient peu des PNIA au moment de décider de leurs choix d'investissement. Ils financent les activités dont ils espèrent un bon retour sur investissement sans se préoccuper des actions qui restent à financer dans les PNIA.

Un autre facteur limitant des PNIA est qu'ils ne permettent pas, en l'état actuel, de comptabiliser ex-post les dépenses effectivement réalisées. Malgré la mise en place de dispositifs de suivi des dépenses, généralement à l'initiative de partenaires techniques et financiers (tels que le Regional Strategic Analysis and Knowledge Support System [ReSAKSS] de l' International Food Policy Research Institute [IFPRI]-USAID et le programme Suivi et analyse des politiques agricoles et alimentaires [SAPAA] de la Food and Agriculture Organization of the United Nations [FAO]), l'information sur l'origine et surtout sur la destination des flux reste incomplète, voire très lacunaire, pour certains acteurs. Le suivi des financements reste essentiellement centré sur les financements publics (bailleurs de fonds et contreparties nationales) et les investissements privés restent assez opaques, non seulement en montants mais surtout en ce qui concerne leur destination. Il est significatif de constater l'absence de tableaux de bord de suivi de l'investissement privé (investissements directs étrangers, portefeuille, via les transferts de revenus, des institutions de microfinance, etc.) au sein des différentes administrations. Il n'est donc pas possible de calculer le niveau de couverture des besoins de financement pour chaque ligne de programme et de sous-programme des différents PNIA. Cela limite fortement la capacité de pilotage et l'éventuelle réorientation des moyens vers les filières identifiées comme prioritaires et néanmoins sous-financées. En conséquence, il existe un risque élevé de sur-financement de certains programmes et de sous-financement d'autres.

Dans la lignée de la Nouvelle alliance (NASAN), les appels au renforcement du dialogue entre acteurs publics et privés se sont multipliés, dans le but de mettre en place des mécanismes de cofinancement et de partenariat public-privé (PPP). Le décalage est toutefois frappant entre ce nouveau discours dominant et la réalité. En dépit de montants d'investissements privés importants dans le secteur agricole de chaque pays, particulièrement en Côte d'Ivoire et au Ghana, le niveau d'articulation avec les financements publics reste assez faible. La situation pourrait toutefois changer dans les prochaines années, au vu des diverses initiatives prises récemment par les pouvoirs publics. Ainsi, le gouvernement du Sénégal a créé depuis 2013 de nouvelles structures publiques ou parapubliques (Fonds souverain d'investissements stratégiques
[FONSIS], Fonds de garantie des investissements prioritaires [FONGIP], Banque nationale pour le développement économique [BNDE]) destinées à encourager les investissements du secteur privé, mais le phénomène est encore trop récent pour que l'impact de ces nouvelles structures soit d'ores et déjà tangible.

Les conditions de mise en place de synergies public-privé ne semblent actuellement pas suffisamment réunies pour permettre leur développement à une échelle qui impacte réellement le secteur. Les finalités poursuivies par chaque type d'acteur sont trop divergentes pour entraîner une adhésion, ne serait-ce que minimale, à des objectifs communs de développement du secteur. Les investisseurs privés affichent certes des préoccupations en termes de durabilité, de responsabilité sociale, de réponses aux besoins du marché du travail, mais leur objectif principal reste la rentabilité de leur capital investi. Ils ne sauraient donc porter et assumer des préoccupations d'intérêt général telles que la réduction de l'incidence de la pauvreté ou la réduction de l'insécurité alimentaire, ni adhérer à l'effort de couverture des besoins identifiés comme nécessaires pour atteindre ces objectifs. Même si le PNIA identifie des besoins de financement non couverts, le secteur privé ne cherchera pas à les combler spontanément s'il n'existe pas de mécanismes fiscaux ou d'autres mesures incitatives qui l'encouragent à le faire. Or de telles mesures sont jusqu'à présent peu développées. Quelques expériences ont été mises en place, comme dans le cas du Ghana où le gouvernement a instauré une fiscalité incitative en vue d'orienter les investissements étrangers vers les régions les moins développées et vers les filières jugées stratégiques par son PNIA, mais ceux-ci continuent de privilégier pour le moment les régions les plus dynamiques (comme les régions Volta et Greater Accra) et les cultures d'exportation les plus rentables commercialement.

\section{Les oubliés du financement}

La complexification du paysage institutionnel du financement, la multiplication des acteurs et la technicisation croissante des modalités d'intervention, dans un contexte où les États ne parviennent pas à canaliser les investissements vers les cibles désirées, conduit inévitablement au sous-financement de divers pans de la politique agricole. Malgré la priorité souvent affichée d'un développement « inclusif », les services financiers formels demeurent très faiblement accessibles à l'agriculture familiale (Wampfler et al., 2014). Les investisseurs privés ciblent très généralement des structures productives de taille conséquente et peu de dépenses publiques sont spécifiquement ciblées sur les plus petits producteurs. La plupart des programmes publics sont globaux, c'est-à-dire qu'ils sont censés bénéficier à l'ensemble des producteurs, mais en pratique ils sont principalement captés par une minorité de bénéficiaires aisés. C'est notamment le cas pour les subventions aux intrants, qui constituent une part très importante des dépenses publiques agricoles. Diop (2015) souligne que, au Sénégal, les revendeurs sont les premiers bénéficiaires du dispositif de subvention aux intrants, au détriment des producteurs, et que même parmi ces derniers, un fort biais existe dans l'attribution des subventions, en faveur des plus grandes exploitations. Il en va de même pour les programmes 
de vulgarisation agricole, dans lesquels les plus gros agriculteurs accèdent plus facilement aux agents gouvernementaux, ou encore pour les actions de collecte de la production, où les plus petits producteurs attendent parfois pendant des jours sans garantie que leur production soit effectivement achetée. Les programmes agricoles généraux ne bénéficient que faiblement aux agriculteurs familiaux, à moins que des mesures spéciales ne soient prises à cet effet.

L'accès au crédit est un autre domaine inégalitaire, où l'obligation de fournir des garanties exclut d'office une partie des producteurs. De plus, le nouvel objectif de développer les « chaînes de valeur » incite les structures publiques de prêt à cibler les petites et moyennes entreprises agroalimentaires. Les crédits bancaires alloués à l'agriculture se positionnent principalement sur l'aval des filières (commercialisation et transformation), sans doute plus solvable que la production, et l'agriculture familiale y a peu accès. Ainsi, les prêts de la Caisse nationale de crédit agricole du Sénégal (CNCAS) sont ciblés sur les importateurs d'intrants (plus de $40 \%$ ) et sur la commercialisation de l'arachide et du coton (de l'ordre de $35 \%$ ).

D'autres angles morts sont thématiques. Il en est ainsi de la question de la sécurisation foncière, qui est cruciale dans de nombreux pays pour son impact social et son rôle dans l'intensification. Les programmes fonciers ne bénéficient pourtant que de faibles financements, qui plus est, le plus souvent pris en charge par les bailleurs de fonds. De tels programmes n'ont représenté que $1,4 \%$ des dépenses publiques agricoles de la Côte d'Ivoire sur la période 2011-2014. Infrastructures et biens publics sont également considérés comme les parents pauvres du financement de l'agriculture. Ils sont essentiellement financés par les bailleurs, à l'instar de la recherche agricole, principalement appuyée par le Programme de productivité agricole en Afrique de l'Ouest (PPAAO), financé par la Banque mondiale. Traditionnellement positionnés sur la fourniture de biens et services publics que le secteur privé n'aurait pas spontanément financés, faute de rentabilité suffisante à court terme, les bailleurs sont en train de redéployer leurs financements à la recherche d'effets de levier pour mobiliser le maximum de fonds privés. Le nouvel objectif de l'aide publique au développement risque d'être de donner en priorité satisfaction aux besoins du secteur privé afin de déclencher le fameux effet catalytique désiré.

On observe une concentration des financements vers certaines régions et certains produits considérés comme prioritaires au regard des politiques agricoles :

- au Sénégal, le programme triennal d'investissements publics 2015-2017 énonce divers projets prioritaires portant sur les filières à haute valeur ajoutée, les corridors céréaliers et l'agrobusiness (République du Sénégal, 2014). L'objectif d'autosuffisance en riz affiché dans le PRACAS (Ministère de l'Agriculture, 2014) absorbe une large partie des financements, même s'il existe une tendance à la diversification tant géographique (en faveur de la Casamance notamment) que vers d'autres filières, horticoles et maraîchères en particulier. Un rééquilibrage important des subventions aux intrants est en train de s'effectuer au détriment de l'arachide et au profit du riz en premier lieu, mais aussi de certaines cultures vivrières telles que le maïs et le mil (Ministère de l'Agriculture, 2012).
L'investissement privé se positionne volontiers sur la partie aval des filières, transformation et commercialisation ;

- en Côte d'Ivoire, le secteur du café-cacao reste celui qui capte le plus de financements publics, de l'ordre de $36 \%$. Le riz et les périmètres hydro-agricoles captent environ $21 \%$ des dépenses publiques agricoles sur la période 2011-2014, en nette progression par rapport aux 9,5\% de la période antérieure, signe de la volonté du gouvernement de réduire la dépendance alimentaire vis-à-vis de l'extérieur. Les secteurs viviers et coton captent chacun de l'ordre de $8 \%$ des dépenses publiques agricoles. Banane et sucre sont principalement soutenus par l'Union européenne à travers les accords Afrique Caraïbes Pacifique (ACP), tandis que certaines filières agro-industrielles telles que l'hévéa et le palmier à huile sont très peu couvertes sur financement public. Leur financement est essentiellement privé. L'analyse de la répartition régionale des financements publics montre des hétérogénéités : les régions des Savanes et du N'Zi-Comoé reçoivent les financements les plus importants ; mais ce sont les régions des Lacs et du Moyen-Comoé qui reçoivent le plus de dépenses publiques par habitant ;

- enfin, dans le cas du Ghana, le secteur cacaoyer est celui qui capte la plupart des financements. La filière cacao, entièrement gérée par l'État ghanéen, représente en moyenne $86 \%$ des dépenses agricoles du gouvernement. Le reste des dépenses publiques agricoles est majoritairement concentré sur le riz, l'hévéa et l'huile de palme. Les investissements étrangers sont plutôt dirigés vers le développement des filières jatropha et de l'horticulture ; les entreprises nationales investissent principalement, sur crédits bancaires, dans la filière cacao.

\section{Conclusion}

Le paysage institutionnel du financement connaît actuellement une mutation profonde, matérialisée par la création récente de nombreuses structures publiques ou parapubliques et la mise en place de montages financiers destinés à favoriser les investissements privés. L'apparition de nouveaux acteurs et de nouveaux mécanismes de financement complexifie la situation et accentue le sentiment d'un déficit de pilotage stratégique. Dès lors, on observe un décalage croissant entre l'impératif de financement des grands enjeux du développement agricole (modèle d'intensification, emploi des jeunes, aménagement du territoire) et la manière dont le secteur agricole est effectivement financé. Chaque acteur joue sa partition en fonction de ses intérêts propres et aucun mécanisme ne permet d'assurer que certains besoins prioritaires seront bien couverts. Les institutions nationales sont généralement trop faibles pour tenir ce rôle de pilotage, du fait de la diminution des capacités d'analyse dans les différents pays, de la faiblesse des capacités de la recherche et de la formation, en un mot du fait du déficit d'investissement dans le capital humain (BAD et al., 2015). Dans ce contexte, le PNIA n'est pas en mesure de jouer le rôle attendu de cadre de référence et d'orientation des investissements agricoles.

Les disparités de financement du secteur agricole, déjà marquées par le passé, s'en trouvent exacerbées. L'apparition ou la montée en gamme de nouveaux instruments tels que les 
fonds de garantie, les assurances agricoles, l'intermédiation bancaire et non bancaire, le financement par fonds compétitifs, ne touche qu'une partie des agriculteurs, correspondant à « l'idéal-type » de l'entrepreneur agricole qui doit déjà être en partie intégré au marché, être déjà dans un Groupement d'intérêt économique (GIE) et donc posséder déjà plusieurs actifs (terre, capital, capital humain). L'accroissement global des montants de financement du secteur agricole cache mal le fait que certains domaines ou types d'acteurs risquent d'être les grands perdants de cette redistribution des cartes. La question du financement durable des biens et services publics, mais aussi de l'agriculture familiale est clairement posée. Il y a un risque évident de marginalisation et d'exclusion sans retour des oubliés du financement.

\section{Références}

Afrique contemporaine. 2011. Investissements agricoles en Afrique ; les migrants dans les villes postcoloniales d'Afrique centrale.Afr Contemp (237), 174 p. Available from https://www.cairn.info/ revue-afrique-contemporaine-2011-1.htm

BAD, OCDE, PNUD. 2015. Commission européenne.Perspectives économiques en Afrique. In: Développement territorial et inclusion spatiale. Paris: Éditions OCDE, Available from http://www.africane conomicoutlook.org/fr

Brochard V. 2015. Le financement du développement agricole au Ghana (2008-2013). Dakar: Études du Hub Rural, 82 p.

Diop AN. 2015. Revue des dépenses publiques de l'agriculture au Sénégal. Revue réalisée pour la Délégation de l'Union européenne, $76 \mathrm{p}$.

FAO. 2012. Investir dans l'agriculture pour un avenir meilleur. In : Rapport sur la situation internationale de l'alimentation et de l'agriculture. Rome : FAO, 202 p. Disponible sur http://www.fao. org/docrep/017/i3028f/i3028f.pdf.

Gabas J-J, Ribier V. 2015. Le financement du développement agricole au Sénégal (2008-2013). Dakar : Études du Hub Rural, 80 p.

IPAR, 2010. Analyse des plans d'investissement agricole ECOWAP/ PDDAA. Les cas du Sénégal, du Mali et de la CEDEAO. 60 p. Disponible sur http://www.ipar.sn/IMG/pdf/Analyse_Plans_Inves tissements_IPAR.pdf.
Ministère de l'Agriculture, République du Sénégal. 2012. Revue diagnostique des dépenses publiques de base dans le secteur agricole. In : Rapport final, 27 avril 2012, Programme Strengthening National Comprehensive Agricultural Public Expenditure in SubSaharian Africa. Banque mondiale et USAID, 155 p. Disponible sur http://documents.worldbank.org/curated/en/221531468007847217/ pdf/831640WP0FRENC00Box379886B00PUBLIC0.pdf.

Ministère de l'Agriculture, République du Sénégal. 2014. Programme de relance et d'accélération de la cadence de l'agriculture sénégalaise (PRACAS), $112 \mathrm{p}$. Disponible sur http://p4arm.org/ app/uploads/2015/02/PRACAS-version-finale.pdf.

Orliange P, de Milly H. 2015. Financement du développement : le public a-t-il encore un rôle à jouer ? In : Issue Brief $n^{\circ} 5 / 15$. Paris : IDDRI. Disponible sur http://www.iddri.org/Publications/Finance ment-du-developpement-le-public-a-t-il-encore-un-role-a-jouer.

OXFAM. 2015. L'ECOWAP, une politique morcelée. Document d'information OXFAM, novembre 2015, 28 p. Disponible sur https://www.oxfam.org/sites/www.oxfam.org/files/file_attach ments/bp-ecowap-fragmented-policy-131115-fr_.pdf.

République de Côte d'Ivoire. 2010. Programme national d'investissement agricole (2010-2015), 118 p. Disponible sur https://www. grain.org/attachments/2957/download.

République du Sénégal. 2010. Programme national d'investissement agricole. Plan d'investissement pour la période 2011-2015. République du Sénégal : CEDEAO, NEPAD, 80 p. Disponible sur https://www.ipar.sn/IMG/pdf/senegal_caadp_post-compact_a gricultural_sector_investment_plan.pdf.

République du Sénégal, Ministère de l'Économie, des Finances et du Plan. 2014. Programme triennal d'investissements publics 2015-2017, 215 p. Disponible sur http:/www.finances.gouv.sn/ images/yootheme/demo/PTIP\%202015-2017.pdf.

Varlet F. 2015. Le financement du développement agricole en Côte d'Ivoire (2008-2013). Dakar : Études du Hub Rural.

Wampfler B, Lesaffre D, Doligez F. 2014. Le financement de l'agriculture en Afrique de l'Ouest : enjeux et perspectives pour les organisations de producteurs agricoles. In : Destrait F, ed. Réponses des organisations paysannes aux besoins de financement des exploitations familiales en Afrique de l'Ouest. Étude SOS Faim, $\mathrm{n}^{\mathrm{o}}$ 9, pp. 10-113. Disponible sur https://www.sosfaim.be/publica tion/reponses-des-organisations-paysannes-aux-besoins-de-finance ment-des-exploitations-familiales-en-afrique-de-louest/.

Citation de l'article : Ribier V, Gabas J-J. 2016. Vers une accentuation des disparités dans le financement de l'agriculture en Afrique de l'Ouest? Cah. Agric. 25: 65007. 